\title{
Prevalence of Obesity and Incidence of Obesity-Related Comorbidities in Koreans Based on National Health Insurance Service Health Checkup Data 2006-2015 (J Obes Metab Syndr 2018;27:46-52)
}

Received July 26, 2018

Reviewed August 20, 2018

Accepted September 3, 2018

${ }^{*}$ Corresponding author

Jin-Hwan Yoon

(iD)

https://orcid.org/0000-0001-9026-6416

Department of Sports Science, Hannam University, 70 Hannam-ro, Daedeok-gu, Daejeon 34430, Korea

Tel: +82-42-629-7990

Fax: +82-42-629-8402

E-mail:yoonjh@hnu.kr

Jin-Hwan Yoon*

Department of Sports Science, Hannam University, Daejeon, Korea

Seo et al. ${ }^{1}$ analyzed the Korean citizen National Health Insurance Service Database (NHIS DB), for which registration is mandatory among Korean citizens, and identified obesity-derived pathogenesis based on the standardization of body mass index (BMI), which was over $25.0 \mathrm{~kg} / \mathrm{m}^{2}$ for all sex, according to the definition of the Asia-Pacific criteria of the World Health Organization guideline ${ }^{2}$, and abdominal obesity greater than $90 \mathrm{~cm}$ and $85 \mathrm{~cm}$ for men and women, respectively, based on the definition of the Korean Society for the Study of Obesity.

However, obesity-related diseases reported by the National Statistical Office (NSO, http://kostat.go.kr) indicated that one out of three individuals has obesity-related metabolic syndrome, which is associated with chronic diseases such as cancer, diabetes, and cardiopathic-related diseases. The NSO reported that cancer is ranked as the primary cause of death in both sexes. Hypertension is ranked 10 th for men and seventh for women and diabetes ranked fifth for men versus fourth for women.

Interestingly, obesity-related metabolic diseases increase in prevalence and associated complications, at and after middle-age. The obesity (A) and abdominal obesity (B) portions stated in Fig. 2 of
Ref. 1 show a reverse change in patterns after the age of 60 years in both sexes. However, the incidence of obesity and abdominal obesity varies; BMI-based obesity indicates a decrease of obesity incidence, while abdominal obesity increased in both sexes after 60 years of age.

The incidence of obesity-related chronic diseases is likely increased in the elderly because of the frail health caused by the lack/ loss of physical fitness; therefore, abdominal obesity should be used as an indicator for this population, rather than the BMI-based obesity index. Muscle mass independent BMI could also contribute to inaccurate measurement values which further supports this recommendation. Muscle mass in body composition affects the individual health state, compared with just the BMI value, therefore abdominal obesity is a more accurate indicator. ${ }^{3}$ For elderly patients, it is important to consider conditions, such as frailty, which is described as follows, frailty is at least three of the following five conditions, including weight loss, lack of energy, impotence, decreased walking speed, and a decrease of physical activity. In addition, the frailty is closely related to physical inactivity which is caused by the lack/loss of physical fitness. 
The concept of frailty and physical fitness is not limited to the elderly, but can also be applied for children. Hanifah et al. ${ }^{4}$ reported that waist circumference (abdominal obesity) rather than BMI is associated with physical fitness in children and adolescents, which emphasizes that abdominal obesity highlights the relationship between obesity and physical activities.

Umpierre et al. ${ }^{5}$ conducted a meta-analysis study on the effects of an intervention that included more than 12 weeks of exercise in patients with diabetes. In this study, the group that completed resistance training exercises, oxidative exercise, and resistance training with oxidative exercise lowered their glycated hemoglobin levels by $0.57 \%, 0.73 \%$, and $0.51 \%$, respectively. Metformin, currently used as a medication for diabetes, decreases the glycated hemoglobin level by $1.5 \%-2 \%$, which indicates a better effect of exercise compared, to medical treatment. The results of this analysis suggest that physical activities can be remarkably effective interventions for obesity-related diseases.

Another study reported that the relationship between obesity and breast cancer at the molecular level included inflammatory-related factors such as tumor necrosis factor-alpha, interleukin 6, and other factors are proportionally increased as disease progression occurred and as obesity increased in cancer patients. ${ }^{6,7}$ Obese patients are more likely to have comorbidities such as hypertension and cardiopathy, and are also more likely to have specific cancers including colon, prostate, breast, and ovarian cancers. ${ }^{8,9}$

The study by Seo et al. ${ }^{1}$ emphasized the essential need for policy that encourages physical activities to control obesity in public health measures because exercise-related prognostic interventions can effectively relieve many obesity-related side effects. Therefore, to provide effective interventions and guidance, public health measures should encourage voluntary physical activity to reduce the risk of and prevalence of obesity. This is an important component of daily health and can impact the etiology of obesity and various chronic lethal diseases such as cancer, diabetes, and hypertension, which are major causes of the death in Korea. Future public health measures should consider this and develop additional evidencebased guidelines for adjusting health and promoting exercise. In addition, it is important to provide reasonable and optimized threshold points for obesity-related parameters by using receiver operative characteristic curve analysis that are applied to current is- sues, as discussed in this article, including exercise parameters. ${ }^{10}$

In conclusion, future health guidance should include additional guidelines targeting obesity. Based on the information in the population-wide registered NHIS DB, standards can be developed that can provide prognostic intervention programs based on specific population data. Specific guidance for activities, such as encouraging physical activity, will be important for improving outcomes for obesity and obesity-derived chronic diseases.

\section{CONFLICTS OF INTEREST}

The author declares no conflict of interest.

\section{REFERENCES}

1. Seo MH, Kim YH, Han K, Jung JH, Park YG, Lee SS, et al. Prevalence of obesity and incidence of obesity-related comorbidities in Koreans based on National Health Insurance Service Health Checkup data 2006-2015. J Obes Metab Syndr 2018;27:46-52.

2. World Health Organization; Regional Office for the Western Pacific. The Asia-Pacific perspective: redefining obesity and its treatment. Sydney: Health Communications Australia; 2000.

3. Rothman KJ. BMI-related errors in the measurement of obesity. Int J Obes (Lond) 2008;32 Suppl 3:S56-9.

4. Hanifah RA, Majid HA, Jalaludin MY, Al-Sadat N, Murray LJ, Cantwell M, et al. Fitness level and body composition indices: cross-sectional study among Malaysian adolescent. BMC Public Health 2014;14 Suppl 3:S5.

5. Umpierre D, Ribeiro PA, Kramer CK, Leitão CB, Zucatti AT, Azevedo MJ, et al. Physical activity advice only or structured exercise training and association with $\mathrm{HbAlc}$ levels in type 2 diabetes: a systematic review and meta-analysis. JAMA 2011; 305:1790-9.

6. Chlebowski RT, Aiello E, McTiernan A. Weight loss in breast cancer patient management. J Clin Oncol 2002;20:1128-43.

7. Pierce BL, Ballard-Barbash R, Bernstein L, Baumgartner RN, Neuhouser ML, Wener MH, et al. Elevated biomarkers of inflammation are associated with reduced survival among breast cancer patients. J Clin Oncol 2009;27:3437-44. 
8. Everett E, Tamimi H, Greer B, Swisher E, Paley P, Mandel L, et al. The effect of body mass index on clinical/pathologic features, surgical morbidity, and outcome in patients with endometrial cancer. Gynecol Oncol 2003;90:150-7.

9. von Gruenigen VE, Courneya KS, Gibbons HE, Kavanagh MB, Waggoner SE, Lerner E. Feasibility and effectiveness of a lifestyle intervention program in obese endometrial cancer pa- tients: a randomized trial. Gynecol Oncol 2008;109:19-26.

10. Jee H, Lee HD, Lee SY. Evidence-based cutoff threshold values from receiver operating characteristic curve analysis for knee osteoarthritis in the 50-year-old Korean population: analysis of big data from the National Health Insurance sharing service. Biomed Res Int 2018;2018:2013671. 